Supporting Information for

\title{
Isomagnetophoresis to Discriminate Subtle Difference in Magnetic Susceptibility
}

\author{
Joo H. Kang, Sungyoung Choi, Wonhye Lee and Je-Kyun Park* \\ Department of Bio and Brain Engineering, Korea Advanced Institute of Science and Technology \\ (KAIST), Daejeon 305-701, Korea
}

* Corresponding author. Je-Kyun Park, Fax: +82-42-869-4310; Tel: +82-42-869-4315. E-mail: jekyun@kaist.ac.kr (J.-K. Park).

\section{Table of Contents:}

1. Analytical model for isomagnetophoresis

2. Microfabrication details

3. Experimental setup and results

Figures S1 S10

Table S1 


\section{Analytical model for isomagnetophoresis}

\section{Analytical model equation}

For theoretical verification, we established an analytical model composing several functions of magnetic drag velocity, $V_{\mathrm{x}}(t, x)$, magnetic susceptibility gradient, $K(t, x)$, magnetic flux density gradient $B(x)$ and particle velocity driven by the parabolic flow profile, $V_{\mathrm{y}}(x) . K(t, x)$ was calculated by the concentration gradient, $C(t, x)$ (Equation 3 ), of each solution in accordance with Wiedemann's additivity law (Equation 5).

$$
\begin{gathered}
V_{x}(t, x)=-\frac{2 R^{2}\left(\chi_{p}-K(t, x)\right) B(x)}{9 \mu_{0} \eta} \\
B(x)=-2.466(100-x)+378.68 \\
C(t, x)=\frac{1}{2} C_{0} \sum_{n=-\infty}^{\infty}\left\{\operatorname{erf} \frac{h+2 n w-x}{2 \sqrt{D t}}+\operatorname{erf} \frac{h-2 n w+x}{2 \sqrt{D t}}\right\} \\
V_{y}(x)=\frac{3}{2} v_{0}\left(1-\frac{4(50-x)^{2}}{w^{2}}\right) \\
\chi(\text { mixture })=\sum_{i=1}^{N} V_{i} \chi_{i} / \sum_{i=1}^{N} V_{i} \\
K(t, x)=C_{G d-D T P A}(t, x) \chi_{G d-D T P A}+\left(1-C_{G d-D T P A}(t, x)\right) \chi_{D-\text { glucose }}
\end{gathered}
$$

where $V_{\mathrm{x}}(\mathrm{t}, \mathrm{x})$ is the magnetophoretic velocity at time, $t(\mathrm{~s})$ at position of $x, R$ radius of a particle (about $7.5 \mu \mathrm{m}), \chi_{\mathrm{p}}$ and $K(t, x)$ volumetric magnetic susceptibility of a particle and fluid, respectively, $B(x)$ magnetic flux density gradient $\left(\mathrm{T}^{2} / \mathrm{m}\right), \mu_{0}$ vacuum permeability, $\eta$ dynamic viscosity of fluid ( $\mathrm{Pa} \mathrm{s}), C_{0}$ initial concentration, $h$ width of the initial distribution $(50 \mu \mathrm{m}), w$ width of the channel $(100 \mu \mathrm{m}), D$ diffusion coefficient of Gd-DTPA $\left(2.3 \times 10^{-6} \mathrm{~cm}^{2} / \mathrm{s}\right)^{1}$ and $v_{0}$ average fluid velocity in the channel $(\mathrm{mm} / \mathrm{s})$. The magnetic susceptibility of Gd-DTPA and Dglucose was obtained from the literatures. ${ }^{2,3} \mathrm{We}$ exploited finite element method magnetics (FEMM) to characterize the magnetic flux density gradient across the microchannel, $B(x)$. The magnetic permeability of nickel was obtained from data in the literature. ${ }^{4}$ Because the microfluidic channel was placed near the edge of a permanent magnet $(\mathrm{NdFeB} 3550 \mathrm{~mm} \times 25$ $\mathrm{mm} \times 10 \mathrm{~mm}$; Magtopia, Korea) and the nickel microstructures were positioned between the permanent magnet and the microfluidic channel, it was hard to calculate the magnetic flux distribution around the microchannels using a numerical equation. Fortunately, the width of the microchannel is enough narrow $(100 \mu \mathrm{m})$ to roughly assume that the magnetic flux density gradient has a linear relationship with the cross sectional distance of the microchannel. Therefore, we made use of Equation (2) in estimation of the magnetophoretic velocity, $V_{\mathrm{x}}(t, x)$.

\section{Prediction of the particle traces under the magnetic field}

Combining the equation stated above, we predicted the particle traces in conventional magnetophoresis and new isomagnetophoresis. In the analytical model, we considered the microparticle as a point in a Cartesian coordinate system assuming that all parts of a microsphere (polymer particle) are exerted by the uniform magnetic force. We iterated the composite function $\left(V_{\mathrm{x}}\right.$ and $V_{\mathrm{y}}$ ) by increasing the time (from $t=0$ ) until the y reaches $11 \mathrm{~mm}$ (microchannel distance). 

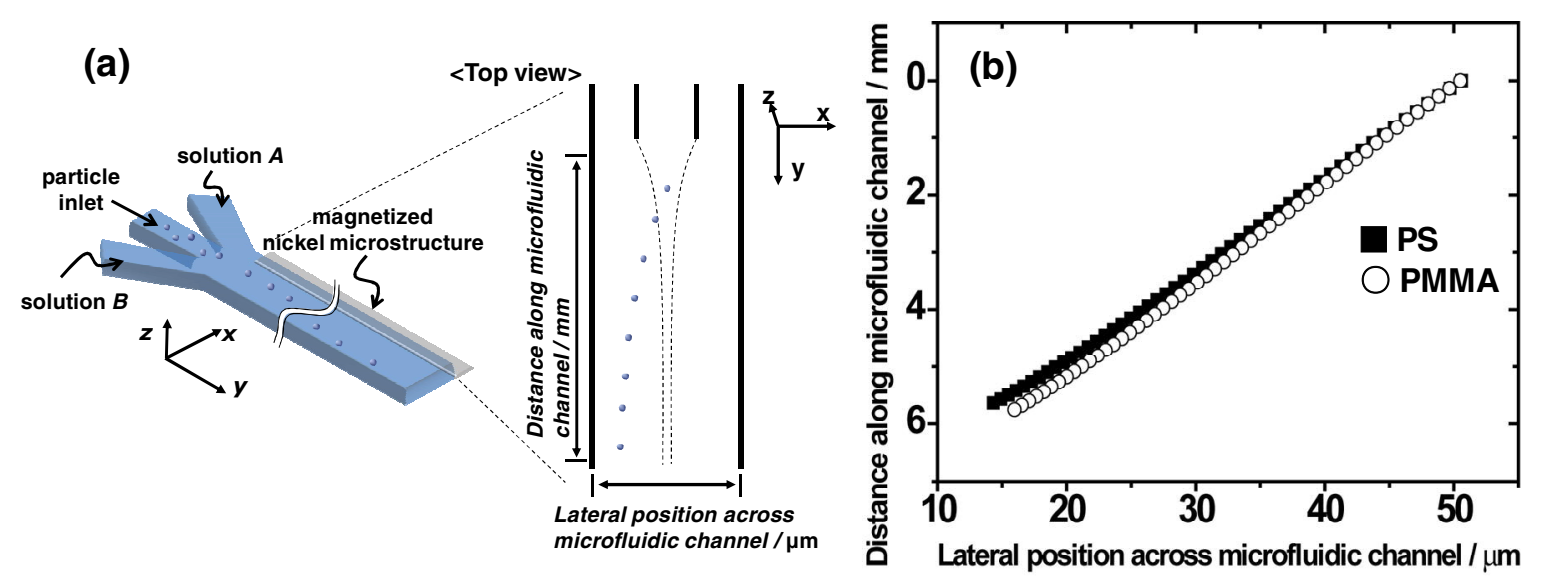

Figure S1. Theoretical estimation of the microparticle displacement in the microfluidic channels of (a) under conventional magnetophoresis. The particle traces of the PS and PMMA particles are plotted in (b) supporting the experimental results as described in Figure 2 and Table S1. The plot presents the theoretical particle traces without the particle size deviation. Considering the practical particle size deviation in Table S1, the lateral positions of PS and PMMA particles in the outlet port become indiscernible.

Table S1. Particle properties and measured profiles of magnetophoresis and isomagnetophoresis

\begin{tabular}{|c|c|c|c|c|c|c|c|c|c|}
\hline & \multirow[b]{2}{*}{$\begin{array}{l}\text { Particle size } \\
\quad(\mu \mathrm{m})\end{array}$} & \multicolumn{3}{|c|}{ Magnetophoresis } & \multicolumn{3}{|c|}{ Isomagnetophoresis } & \multicolumn{2}{|c|}{$\chi\left(10^{-6}\right)$} \\
\hline & & $\begin{array}{l}\text { Lateral } \\
\text { position } \\
\text { (average) }\end{array}$ & $\begin{array}{l}\text { Standard } \\
\text { deviation }\end{array}$ & $\begin{array}{l}\text { Particle } \\
\text { number }\end{array}$ & $\begin{array}{l}\text { Lateral } \\
\text { position } \\
\text { (average) }\end{array}$ & $\begin{array}{l}\text { Standard } \\
\text { deviation }\end{array}$ & $\begin{array}{l}\text { Particle } \\
\text { number }\end{array}$ & Reference & Estimated \\
\hline PS & $14.88 \pm 0.20^{a}$ & 18.52 & 1.58 & 247 & 17.68 & 1.62 & 576 & $8.24^{c}$ & -8.75 \\
\hline PMMA & $15.00 \pm 0.77^{a}$ & 18.20 & 6.61 & 802 & 30.44 & 3.48 & 406 & - & -2.10 \\
\hline BS & $14.01 \pm 1.00^{b}$ & 38.08 & 8.43 & 832 & 52.13 & 5.33 & 1097 & - & +12.90 \\
\hline
\end{tabular}

${ }^{a}$ measured by Beckman Coulter LS, ${ }^{b}$ Duke Scientific Corp. offered the data measured by NIST, ${ }^{c}$ ref. 6. 


\section{Microfabrication details}

\section{Microfluidic device for isomagnetophoresis}

The microfluidic devices were fabricated by PDMS micromolding processes using a SU8 patterned Si wafer mold as described in Figure S2. The replicated PDMS substrates were bonded with the PDMS-coated slide glass and the nickel microstructure by air plasma treatment. Nickel microstructures for enhancement of magnetic flux density gradient ${ }^{5}$ were prepared by electroplating using THB $151 \mathrm{~N}$ photoresist molds. As shown in Figure S3, the device has three inlets and single outlet. Two inlets for Gd-DTPA and D-glucose employed micropillar arrays for filtration of inevitable dusts in solution. To achieve more stable microfluidic control, we designed serpentine long microfluidic channel for input microfluidic resistance. These fluidic resistances suppress the microfluidic fluctuation caused by a conventional syringe pumping system. Passing through the magnetophoresis region, the particles were detected at the extended outlet channel by a CCD camera.

\section{Microfluidic device for measurement of the concentration gradient of Gd-DTPA and D-} glucose solution

To verify the concentration gradient of Gd-DTPA, we fabricated PDMS microfluidic devices having two inlets for Gd-DTPA and D-glucose and eight-branched-off outlets. The microfluidic device was also prepared by PDMS micromolding processes. The replicated PDMS substrate was bonded with a cleaned slide glass using air plasma treatment. The fabricated device is depicted in Figure S4.

1. Seed metal layer and PR mold pattering

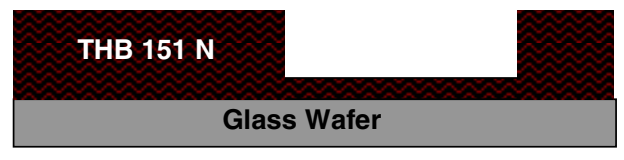

2. Ni electroplating (50 $\mu \mathrm{m}$-thick)

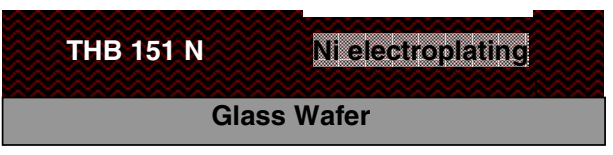

3. PR strip and PDMS coating

\begin{tabular}{|c|}
\hline PDMS \\
\hline Mlass Wafer \\
\hline
\end{tabular}

\section{PR strip and PDMS coating}
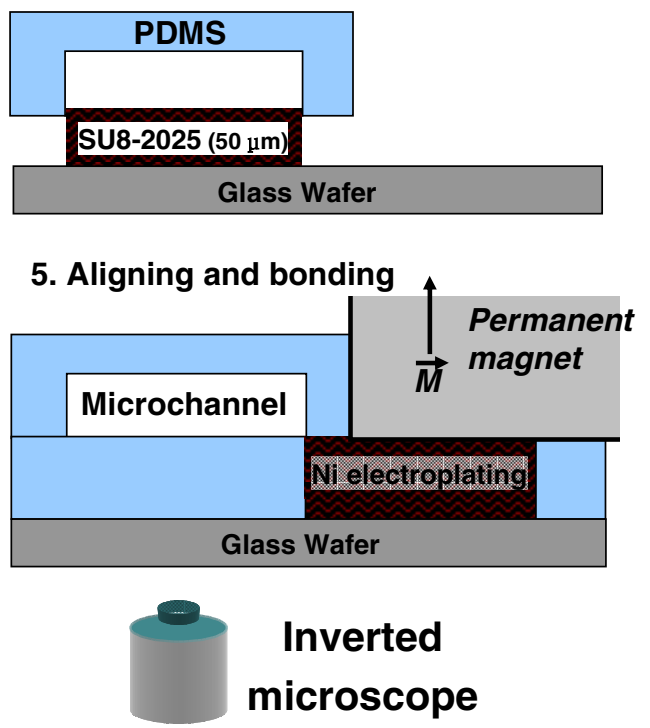

Figure S2. Microfabrication processes and the experimental setup for observation of the particles. 


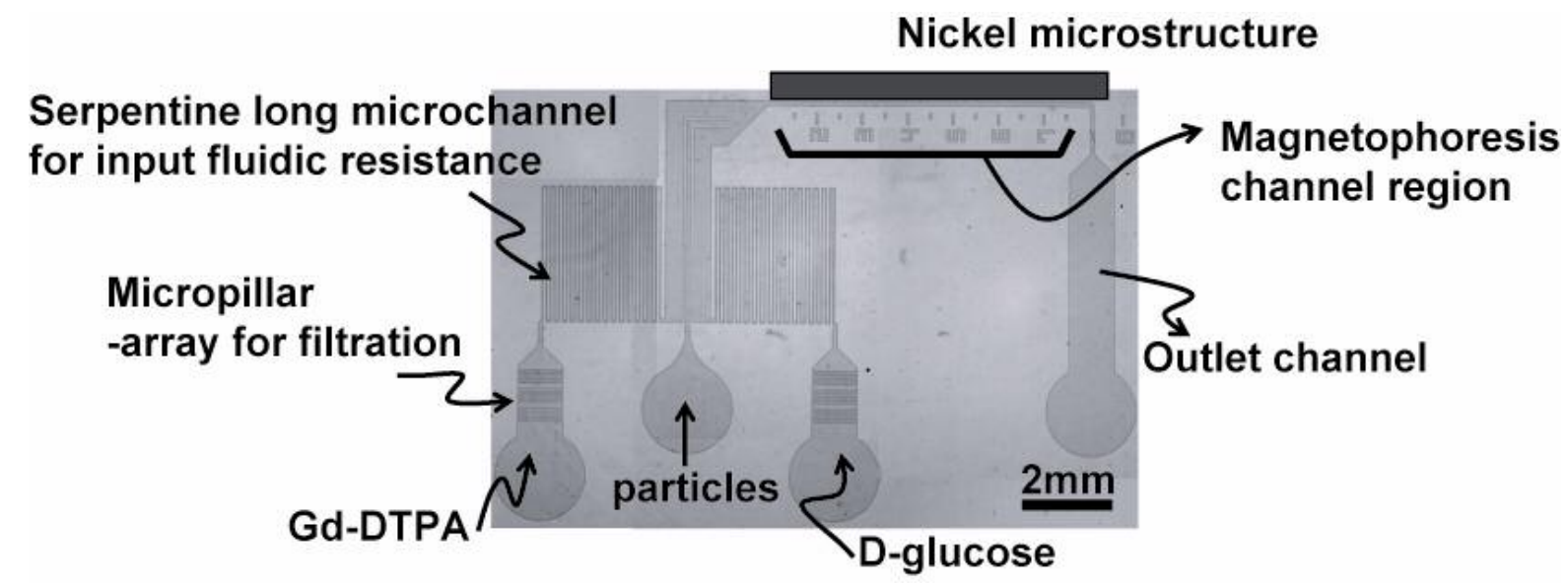

Figure S3. The microscopic image $(\times 10)$ showing the fabricated microfluidic device.

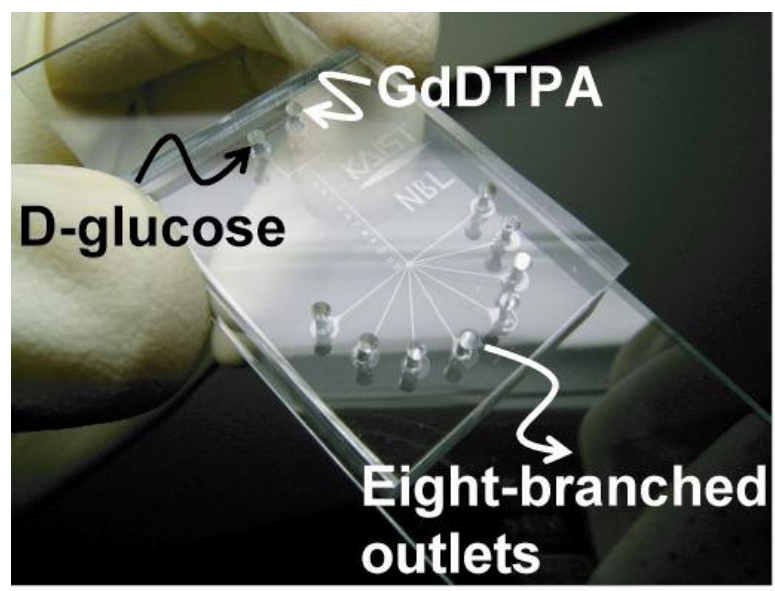

Figure S4. The picture showing the microfluidic device for measuring the concentration profiles of Gd-DTPA and D-glucose solution. 


\section{Experimental setup and results}

\section{Measuring the concentration gradient of Gd-DTPA and D-glucose}

We estimated the gradient profiles of Gd-DTPA and D-glucose using finite element method (FEM) (CFD-ACE+) and an analytical equation (Equation 3). To confirm the estimated concentration gradient profile, we compared that with the experimental results using UV spectroscopy. We measured Gd-DTPA (125 mM) absorbance scanning from $190 \mathrm{~nm}$ to $1800 \mathrm{~nm}$ with reference to distilled water (DIW) (using Shimadzu 1610 PC and Quartz cuvette). As a result, we found that $\mathrm{Gd}-\mathrm{DTPA}$ has a peak at $274.5 \mathrm{~nm}\left(25^{\circ} \mathrm{C}\right)$ (Figure S5). To obtain extinction coefficient based on Beer-Lambert law $(A=\varepsilon \cdot c \cdot l)$, where $A$ is absorbance (a.u.), $\varepsilon$ extinction coefficient, $c$ concentration and $l$ path-length penetrated by light. As presented in Figure S6, we obtained $\varepsilon_{\text {Gd-DTPA }} 1.44 / \mathrm{M} \mathrm{cm}$ (at $274.5 \mathrm{~nm}, 25^{\circ} \mathrm{C}$, ref DIW).

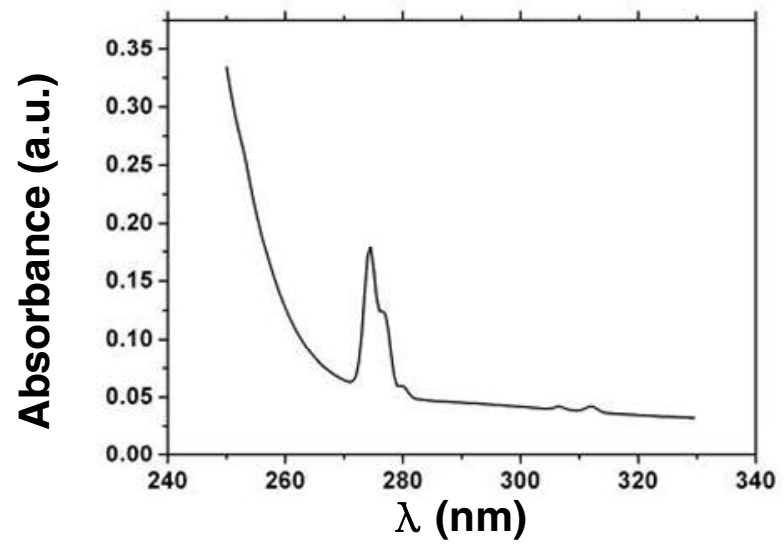

Figure S5. Absorbance profile of Gd-DTPA $(125 \mathrm{mM})$ around UV light. Gd-DTPA has a peak at $274.5 \mathrm{~nm}$.

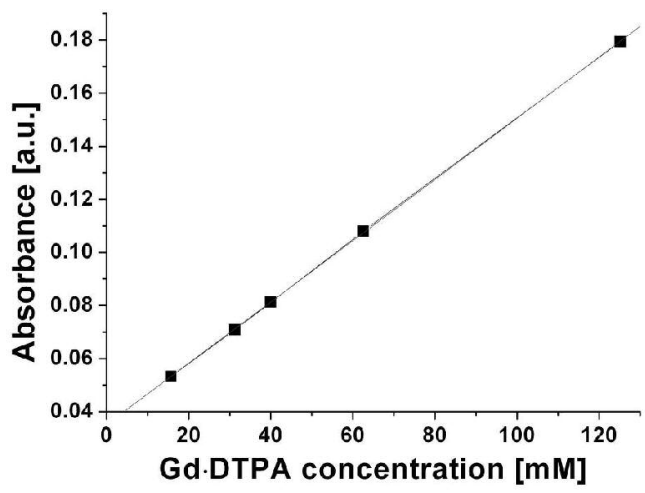

Figure S6. Absorbance of Gd-DTPA at $274.5 \mathrm{~nm}$ according to the concentration. The plotted data provide the extinction coefficient of Gd-DTPA according to the Beer-Lambert relationship. 
To compare the Gd-DTPA / D-glucose concentration profile with that estimated by numerical analysis, we measured the concentration gradient. Using the microfluidic device exhibited in Figure S4 and S7, we collected the samples $(200 \mu \mathrm{L}$, for $40 \mathrm{~h}$ at a flow rate of 0.5 $\mu \mathrm{L} / \mathrm{min}$ ) from the eight-branched-off outlets of the microfluidic channels and they were assessed by UV spectroscopy to obtain the relative concentration of Gd-DTPA with reference to Dglucose at $274.5 \mathrm{~nm}$. Figure S8 presents a correlation between the experimental result, numerical simulation of CFD-ACE + and calculation by the analytical equation. The analytical equation, simulation and experimental result resulted from consideration of one, two and three dimensional mass transportation, respectively, which are governed by diffusion, convection and hyperbolic flow rate through the microchannel. In particular, the hyperbolic profile of the flow rate reduces the diffusion time between the two adjoining laminar flows around the center of the channel so that the experimental results on the gradient profile has a steeper slope of the gradient around the center region of the channel compared to the prediction using the diffusion equation.

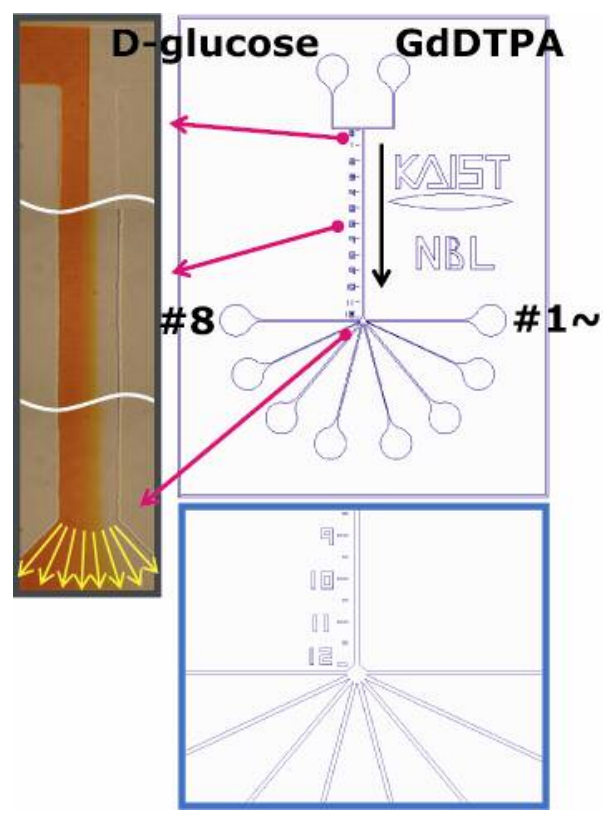

Figure S7. Schematic of the microfluidic device for measuring the concentration profiles of GdDTPA and D-glucose solution. Two solutions (red food dye and DIW in a picture left for the visualized demonstration) are injected into inlets and flow down along the microchannel with diffusion. Eight-branched-off outlets gather the distributed sample solutions. 


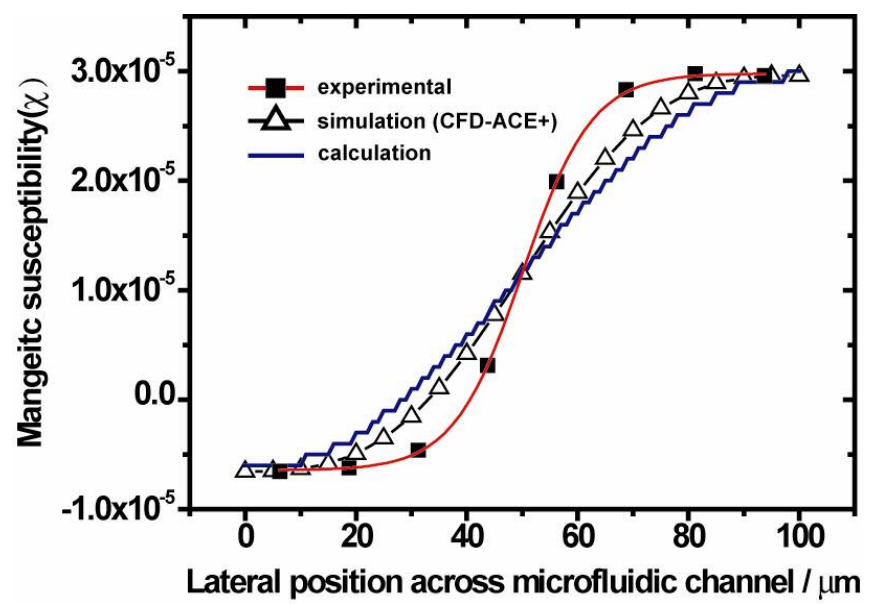

Figure S8. The magnetic susceptibility gradient across the channel at $11 \mathrm{~mm}$ along the microfluidic channel. The magnetic susceptibility was calculated from the concentration profiles using Wiedemann's additivity law (Equation 5).

\section{Dynamic viscosity measurement}

Viscosity of two fluids (Gd-DTPA and D-glucose) and mixtures of them has been measured by a viscometer (Vibro Viscometer SV-10; Malvern Instruments Ltd., UK). The measured viscosity is presented in Figure S9. The sample solutions for the measurement were prepared according to the mixing ratio of two solutions (volume fraction of Gd-DTPA in total volume of solution, $1.0,0.8,0.6,0.4,0.2$ and 0.0 ). The viscosity was recorded for 3 min every $5 \mathrm{~s}$ providing 40 50 data of the measured values and we plotted the averaged viscosity and standard deviation in Figure S9. As presented in the plot, the viscosity varies along the cross-section of the microchannel with the errors less than $5 \%$ from the mean value. Consequently, we used the average dynamic viscosity of $1.33 \times 10^{-3} \mathrm{~Pa} \mathrm{~s}$ (at $23.5^{\circ} \mathrm{C}$ ) in Equation (1). As a reference, we also assessed viscosity of DIW $\left(1.12 \times 10^{-3} \mathrm{~Pa} \mathrm{~s}\right.$, at $\left.23.5^{\circ} \mathrm{C}\right)$, relatively higher than the expected value $\left(\sim 0.90 \times 10^{-3} \mathrm{~Pa} \mathrm{~s}\right.$, at $\left.23^{\circ} \mathrm{C}\right)$ and Gd-DTPA $\left(1.23 \times 10^{-3} \mathrm{~Pa} \mathrm{~s}\right.$, at $\left.23.5^{\circ} \mathrm{C}, 125 \mathrm{mM}\right)$

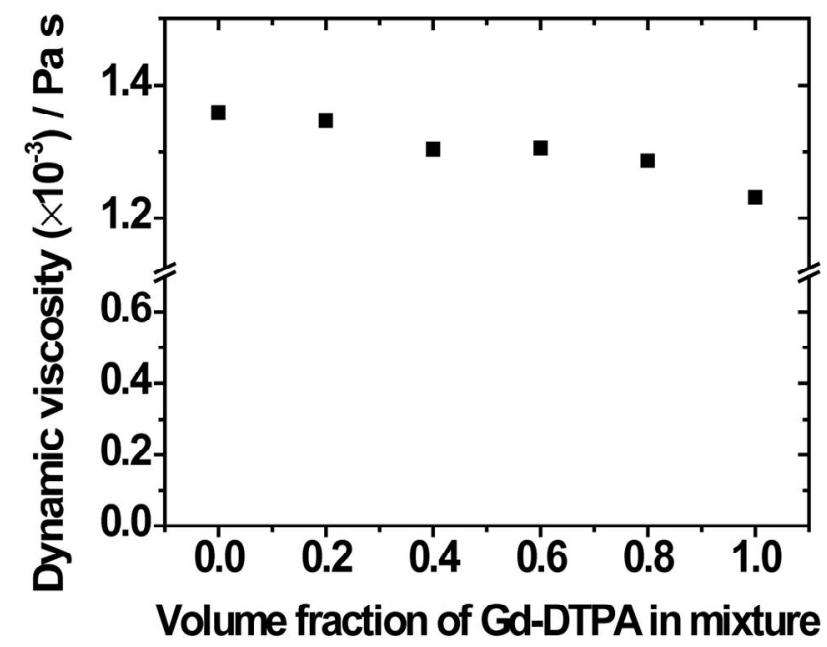

Figure S9. Viscosity measurement data according to the volume fraction of Gd-DTPA in mixture of Gd-DTPA and D-glucose. 


\section{Particle preparation}

PS, PMMA and BS microparticles were purchased from Sigma Aldrich Korea, Bangs Laboratories, Inc. (Fishers, IN) and Duke Science Corporation (Fremont, CA), respectively. PMMA, BS particles were delivered as a powder type so that they were dispersed in Gd-DTPA solution with $0.2 \%$ of Tween ${ }^{\circledR} 20$.

\section{Particle injection and measurement of the lateral position}

All polymer particles used herein were dispersed in Gd-DTPA $(125 \mathrm{mM})$ solution to perform magnetophoresis and isomagnetophoresis. The volumetric flow rate $(1.1 \mu \mathrm{L} / \mathrm{min})$ in magnetophoresis was faster than that $(0.44 \mu \mathrm{L} / \mathrm{min})$ in isomagnetophoresis because the drag velocity driven by magnetophoresis was greater than that by isomagnetophoresis because $\Delta \chi$ between the particles and surrounding solution in magnetophoresis was larger along the microfluidic channel than that in isomagnetophoresis. Therefore, since the particles of PS and PMMA were focused and stood in line at the left side of the outlet channel (Figure S10), we needed to increase the flow rate in magnetophoresis to give the reduced retention time in the microchannel.

The polymer particles in the microfluidic channel were observed by an inverted microscope and images were recorded by a CCD camera for about 30 min every $5 \mathrm{~s}$. The obtained images were converted into binary images and adjusted to the proper threshold value using MATLAB. The detected particle positions were output into $x-y$ coordinates in *.txt file.
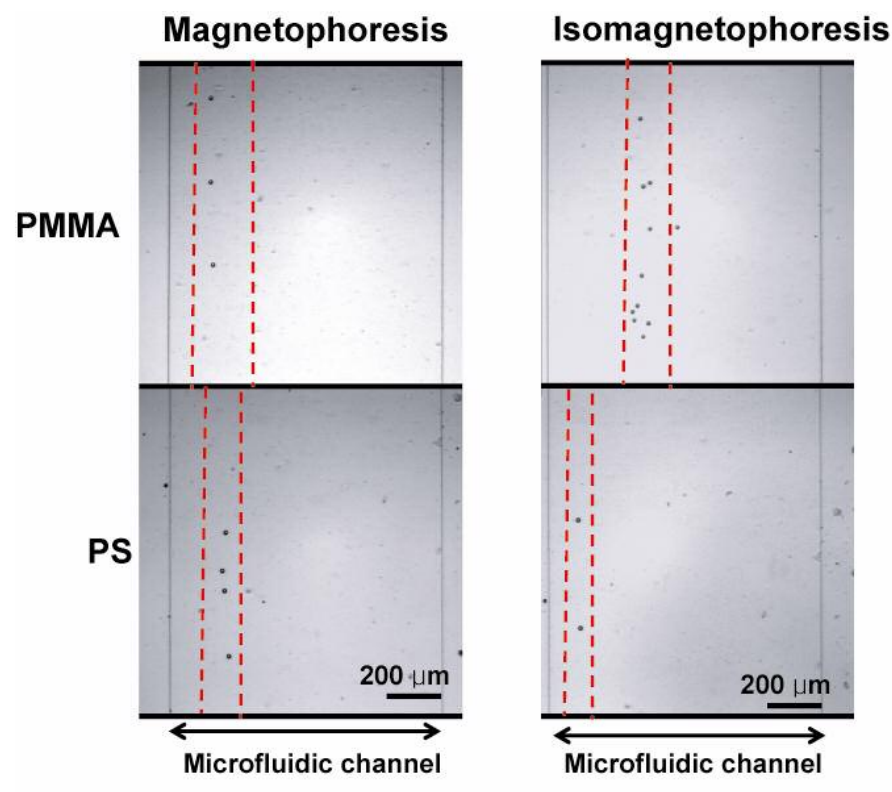

Figure S10. The captured images of PS and PMMA microparticles. The red dashed-lines present the range of the detected lateral position of each type of the particles, where the lateral position of the particles is distinguishable in isomagnetophoresis while it is not discernible in the conventional magnetophoresis. The particle position was measured in the $1000 \mu \mathrm{m}$-extended outlet channel to retard the flow velocity of the particle. 


\section{Complete reference 1}

(1) Haddon, R. C.; Schneemeyer, L. F.; Waszczak, J. V.; Glarum, S. H.; Tycko, R.; Dabbagh, G.; Kortan, A. R.; Muller, A. J.; Mujsce, A. M.; Rosseinsky, M. J. ; Zahurak, S. M.; Makhija, A. V.; Thiel, F. A.; Raghavachari, K.; Cockayne, E.; Elser, V. Nature 1991, 350, 46-47.

\section{References}

(1) Gordon, M. J.; Chu, K. C.; Margaritis, A.; Martin, A. J.; Ethier, C. R.; Rutt, B. K. Biotechnol. Bioeng. 1999, 65, 459-467.

(2) Zhang, H.; Moore, L. R.; Zborowski, M.; Williams, P. S.; Margelc, S.; Chalmers, J. J. Analyst 2005, 130, 514-527.

(3) Winkleman, A.; Gudiksen, K. L.; Ryan, D.; Whitesides, G. M.; Greenfield, D.; Prentiss, M. Appl. Phys. Lett. 2004, 85, 2411-2413.

(4) Suzuki, T.; Baba, H.; Matsumoto, E. Int. J. Appl. Electromag. Mech. 2001, 13, 307-310.

(5) Kang, J. H.; Park, J.-K. Small 2007, 3, 1784-1791.

(6) Watarai, H.; Namba, M. Anal. Sci. 2001, 17, 1233-1236. 